\title{
GWAS and co-expression network combination uncovers multigenes with close linkage effects on the oleic acid content accumulation in Brassica napus
}

Min Yao ${ }^{1 \dagger}$, Mei Guan ${ }^{1+}$, Zhenqian Zhang ${ }^{1}$, Qiuping Zhang ${ }^{1}$, Yixin Cui ${ }^{2}$, Hao Chen ${ }^{1}$, Wei Liu ${ }^{1}$, Habib U. Jan ${ }^{3}$ Kai P. Voss-Fels ${ }^{4}$, Christian R. Werner ${ }^{5}$, Xin He ${ }^{1}$, Zhongsong Liu' ${ }^{1}$, Chunyun Guan ${ }^{1}$, Rod J. Snowdon ${ }^{6}$,

Wei Hua ${ }^{1,7^{*}}$ and Lunwen Qian ${ }^{1 *}$

\begin{abstract}
Background: Strong artificial and natural selection causes the formation of highly conserved haplotypes that harbor agronomically important genes. GWAS combination with haplotype analysis has evolved as an effective method to dissect the genetic architecture of complex traits in crop species.

Results: We used the $60 \mathrm{~K}$ Brassica Infinium SNP array to perform a genome-wide analysis of haplotype blocks associated with oleic acid (C18:1) in rapeseed. Six haplotype regions were identified as significantly associated with oleic acid (C18:1) that mapped to chromosomes A02, A07, A08, C01, C02, and C03. Additionally, whole-genome sequencing of 50 rapeseed accessions revealed three genes (BnmtACP2-A02, BnABCl13-A02 and BnECI1-A02) in the A02 chromosome haplotype region and two genes (BnFAD8-C02 and BnSDP1-C02) in the C02 chromosome haplotype region that were closely linked to oleic acid content phenotypic variation. Moreover, the co-expression network analysis uncovered candidate genes from these two different haplotype regions with potential regulatory interrelationships with oleic acid content accumulation.
\end{abstract}

Conclusions: Our results suggest that several candidate genes are closely linked, which provides us with an opportunity to develop functional haplotype markers for the improvement of the oleic acid content in rapeseed.

Keywords: Oleic acid, GWAS, Haplotype, Co-expression network, Brassica napus

\section{Background}

Oilseed rape (Brassica napus L.) is an allotetraploid species with $2 n=38$ chromosomes and two genomes (AA derived from B. rapa and CC from B. oleracea). It is the most important source of edible vegetable oil and protein-rich meal in China. Rapeseed oil is similar to

\footnotetext{
*Correspondence: huawei@oilcrops.cn; qianlunwen@163.com

${ }^{\dagger}$ Min Yao and Mei Guan contributed equally to this work.

${ }^{1}$ Collaborative Innovation Center of Grain and Oil Crops in South China, Hunan Agricultural University, Changsha 410128, China

Full list of author information is available at the end of the article
}

other vegetable oils. Its fatty acid composition is the key trait involved in its utilization mode and range [1]. The proportions of the three major unsaturated fatty acids in rapeseed oil are $60 \%$ oleic acid [C18:1], 4\% palmitic acid [C16:0] and 2\% stearic acid [C18:0]. Furthermore, oleic acid has been recognized as having health benefits, including effectiveness in reducing overall cholesterol levels, as well as anti-arteriosclerosis and cardiovascular protective effects; therefore, a higher oleic acid content level in seed oil is a desirable trait. Menendez et al. [2] suggested that the anti-cancerous and heart-protective

(c) The Author(s). 2020 Open Access This article is licensed under a Creative Commons Attribution 4.0 International License, which permits use, sharing, adaptation, distribution and reproduction in any medium or format, as long as you give appropriate credit to the original author(s) and the source, provide a link to the Creative Commons licence, and indicate if changes were made. The images or other third party material in this article are included in the article's Creative Commons licence, unless indicated otherwise in a credit line to the material. If material is not included in the article's Creative Commons licence and your intended use is not permitted by statutory regulation or exceeds the permitted use, you will need to obtain permission directly from the copyright holder. To view a copy of this licence, visit http://creativecommons.org/licenses/by/4.0/ The Creative Commons Public Domain Dedication waiver (http://creativecommons.org/publicdomain/zero/1.0/) applies to the data made available in this article, unless otherwise stated in a credit line to the data. 
properties of the Mediterranean diet could also be attributed to 18:1. Pinzi et al. [3] suggested that high oleic acid oil was an ideal raw material for biodiesel production. High oleic acid oil can be heated to a higher temperature without smoking, which makes it more suitable as a cooking oil. Therefore, further improvement of the oleic acid oil content has become a primary goal of rapeseed breeding.

In plants, fatty acid biosynthesis is a very complicated process. Li-Beisson et al. [4] reported more than 120 enzymatic reactions and at least 600 genes were involved in acyl-lipid metabolism process in Arabidopsis. Fatty acid biosynthetic pathways in rapeseed are considered quantitative traits regulated by QTLs. Wang et al. [5] detected 72 individual QTLs and a large number of pairs of epistatic interactions associated with the content of 10 different fatty acids. For the oleic acid (C18:1) content, the major QTLs are mainly distributed across A03, A05, A08, C03 and C08 [6-11]. Since the advent of highly efficient genotyping technologies, the genomewide association study (GWAS) has become the method of choice for dissection of complex plant traits. For example, a GWAS detected more than 100 single nucleotide polymorphisms (SNPs) in significant associations with the oleic acid content on chromosomes A06, A08, A09, C01, C3, C04, C08 and C09 [12]. Guan et al. [13] identified 95 candidate genes involved in fatty acid biosynthesis in the whole genome using a GWAS in combination with RT-qPCR analysis.

Strong selection can cause multigene close linkages in genome regions related to target trait phenotypic variation. Recently, some haplotype-based GWAS detected haplotype regions containing several causal genes related to phenotypic variation in crops. For example, a GWAS performed with the Illumina $90 \mathrm{k}$ SNP Infinium array to check a haplotype region (at 137.1 and $143.5 \mathrm{cM}$ ) that contained candidate genes related to root growth on chromosome 5B in wheat [14]. Qian et al. [15] identified a haplotype carrying candidate genes BnTOC159 and BnaA02g20650D, which were significantly associated with the leaf chlorophyll content on chromosome A02 in rapeseed. A genome-wide haplotype association analysis detected haplotypes containing two candidate genes (glyma12g075700 and glyma12g075600) that showed significant associations with the seed weight and seed yield in soybean [16]. Although the haplotype regions containing several causal genes that are significantly associated with the target trait can be detected, however, little is known if these putative genes have a potential relationship with the haplotype regions.

Gene function can be established through reverse genetic approaches in many crops, and co-expression networks have been shown to be a powerful tool for the rapid prediction of potential functional links between genes. Recent studies have suggested that a GWAS in combination with a co-expression network is a powerful tool to identify genes related to phenotypic variation in maize and Populus $[17,18]$. This method will also permit analysis of relationships among genes with significant associations in a haplotype region.

In our study, we perform a GWAS of haplotype blocks and identify six haplotype blocks carrying causal genes associated with the seed oleic acid content in a diverse B. napus population. In particular, our objectives were to uncover several candidate genes in close linkage due to strong selection and to identify a potential molecular network regulated in the accumulation of oleic acid content by whole-genome sequencing of 50 accessions and gene co-expression. Our results will help in developing functional haplotype makers to further improve oleic acid content in B. napus.

\section{Results \\ Oleic acid content variation and correlations}

In this study, significant variation in the oleic acid content was observed across the diversity panel in three different years in Chongqing. The frequency distribution of the oleic acid content for the three different years is summarized in Fig. 1. In the three environments (years 2013, 2014 and 2015) in Chongqing, the oleic acid contents ranged from 19.83 to 67.83 (\%), 6.49 to 67.20 (\%) and 13.77 to 69.96 (\%), with an average value $( \pm \mathrm{SD})$ of $54.86 \pm 12.1(\%), 55.53 \pm 12.8(\%)$ and $54.74 \pm 11.7(\%)$ and variable coefficients of $22.03,22.99$ and $21.32 \%$, respectively (Table 1). A high broad-sense heritability of $\mathrm{H}^{2}=0.54$ was calculated for the oleic acid content (Additional file 1: Table S1). The oleic acid content showed significant positive correlations across different environments (i.e. years) in Chongqing with a correlation coefficient of 0.42 to 0.72 (Table 1). This observation indicated that the oleic acid content exhibited relatively stable genetic variation in the diversity panel.

\section{Oleic acid content GWAS}

Manhattan plots and quantile-quantile plots describing significant SNP associations with the oleic acid content in the three different environments are shown in Fig. 2. A total of 51 SNPs distributed throughout the genome were detected in an association with oleic acid content using the significance threshold of $-\log _{10}{ }^{(p)}=4$. Candidate regions containing SNPs associated with the oleic acid content were investigated at a high resolution by assaying haplotype blocks $\left(\mathrm{r}^{2}>0.50\right)$ in flanking chromosome segments. Six significantly associated haplotypes were detected on chromosomes A02, A07, A08, C01, $\mathrm{CO} 2$ and C03 (Fig. 3). Details of the SNPs and the candidate genes in the haplotype blocks with significant 

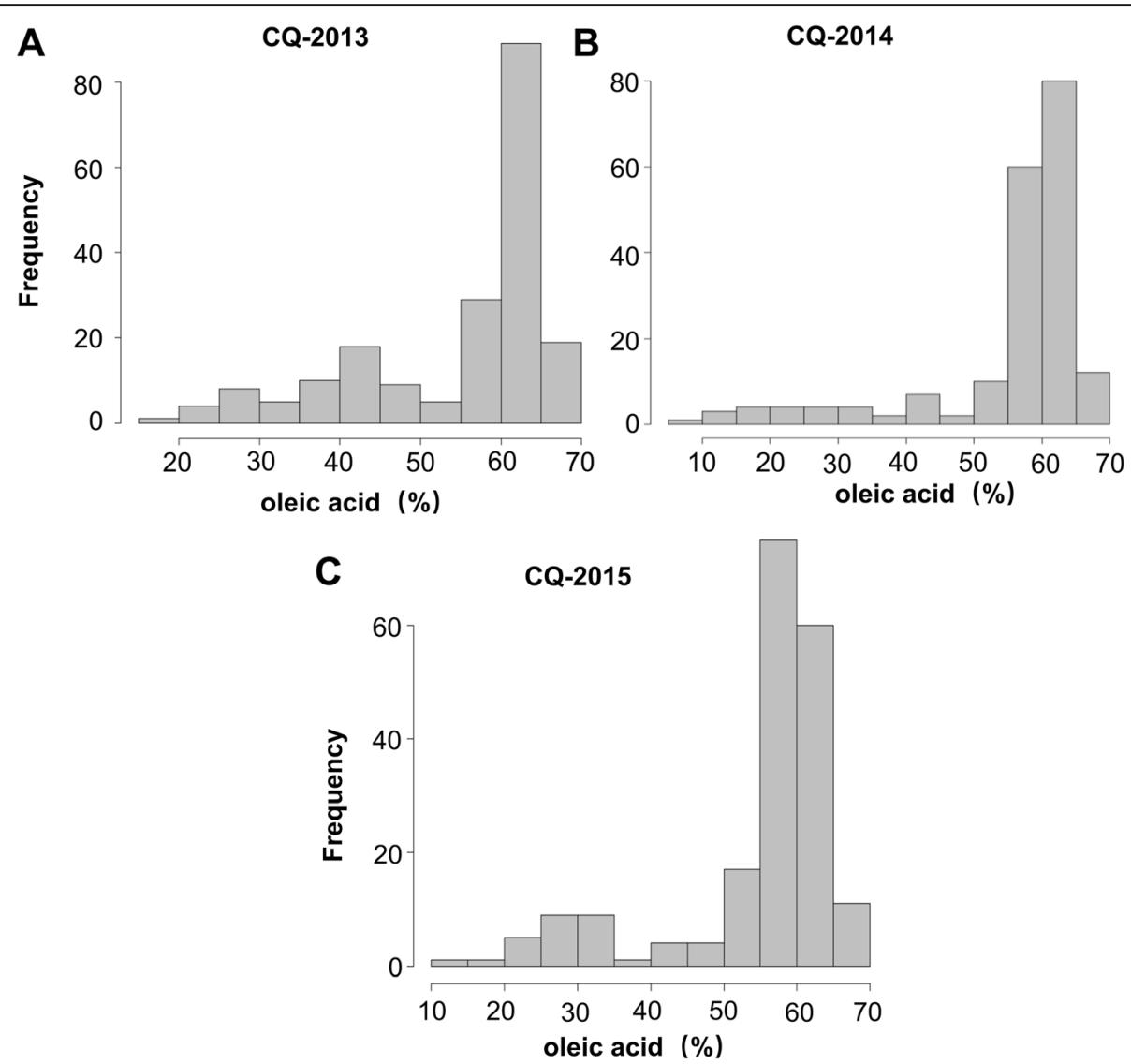

Fig. 1 The frequency distribution of the oleic acid content in the three environments in 203 Chinese semi-winter rapeseed accessions. (a) 2013 in Chongqing (CQ-2013); (b) 2014 in Chongqing (CQ-2014); (c) 2015 in Chongqing (CQ-2015)

associations with oleic acid are shown in Additional file 2: Table S2.

\section{Multiple genes in close linkage with a positive effect on} the accumulation of oleic acid content

On chromosome A02, we identified a haplotype block (6,311,853-6,435,462 bp; A02_Hap) significantly associated with oleic acid content, and containing three $B$. napus orthologues of the Arabidopsis genes MITOCHONDRIAL ACYL CARRIER PROTEIN 2 (BnMTACP2-A02; BnaA02g12050D), ATP-BINDING CASSETTE I13 (BnABCI13-A02; BnaA02g12080D) and DELTA (2)-ENOYL COA ISOMERASE 1 (BnECI1-A02;

Table 1 Phenotypic characteristics of the oleic acid content in 203 Chinese semi-winter rapeseed accessions

\begin{tabular}{lllllll}
\hline Environment & $\begin{array}{l}\text { Min } \\
(\%)\end{array}$ & $\begin{array}{l}\text { Max } \\
(\%)\end{array}$ & $\begin{array}{l}\text { Mean } \pm \text { SD } \\
(\%)\end{array}$ & CV\% & \multicolumn{2}{l}{ Environment } \\
\cline { 6 - 7 } & & & & & CQ-2014 & CQ-2015 \\
\hline CQ-2013 & 19.83 & 67.83 & $54.86 \pm 12.10$ & 22.03 & $0.42^{* * *}$ & $0.55^{* * *}$ \\
CQ-2014 & 6.49 & 67.20 & $55.53 \pm 12.80$ & 22.99 & & $0.72^{* * *}$ \\
CQ-2015 & 13.77 & 69.96 & $54.74 \pm 11.70$ & 21.32 & & \\
\hline
\end{tabular}

CQ Chongqing; $S D$ standard deviation; $C V$ coefficient of variation; ${ }^{* * *} p \leq 0.001$
BnaA02g12140D) in this haplotype region (Fig. 3). mtACP2 encodes a member of the mitochondrial acyl carrier protein (ACP) family that is involved in the fatty acid biosynthesis process, ABCI13 is an ATP-binding cassette $(\mathrm{ABC})$ transporter that is involved in chloroplast lipid import, and ECI1 encodes a peroxisomal delta3, delta2-enoyl CoA isomerase that is involved in unsaturated fatty acid degradation (Additional file 2: Table S2). In addition, whole-genome resequencing of 50 accessions from the same diversity panel was used to further analyze the A02_Hap region. We found that six SNPs were located in these three gene regions, including one in intron 1 of BnMTACP2-A02, two in exon 1 of $\mathrm{BnABCI} 13-\mathrm{A} 02$ and three in exon 1 of the BnECHICA02 (Fig. 4a). This result suggests that these three genes are in close linkage in the A02_Hap region. By comparing the oleic acid content and gene expression levels of the two haplotype alleles in the A02_Hap region, we found that A02_MTACP2+ ABCI13 + ECI1-HAP1 had a higher oleic acid content and that the BnABCI13-A02 and BnMTACP2-A02 genes also showed relatively higher expression levels than A02_MTACP2+ ABCI13 + 


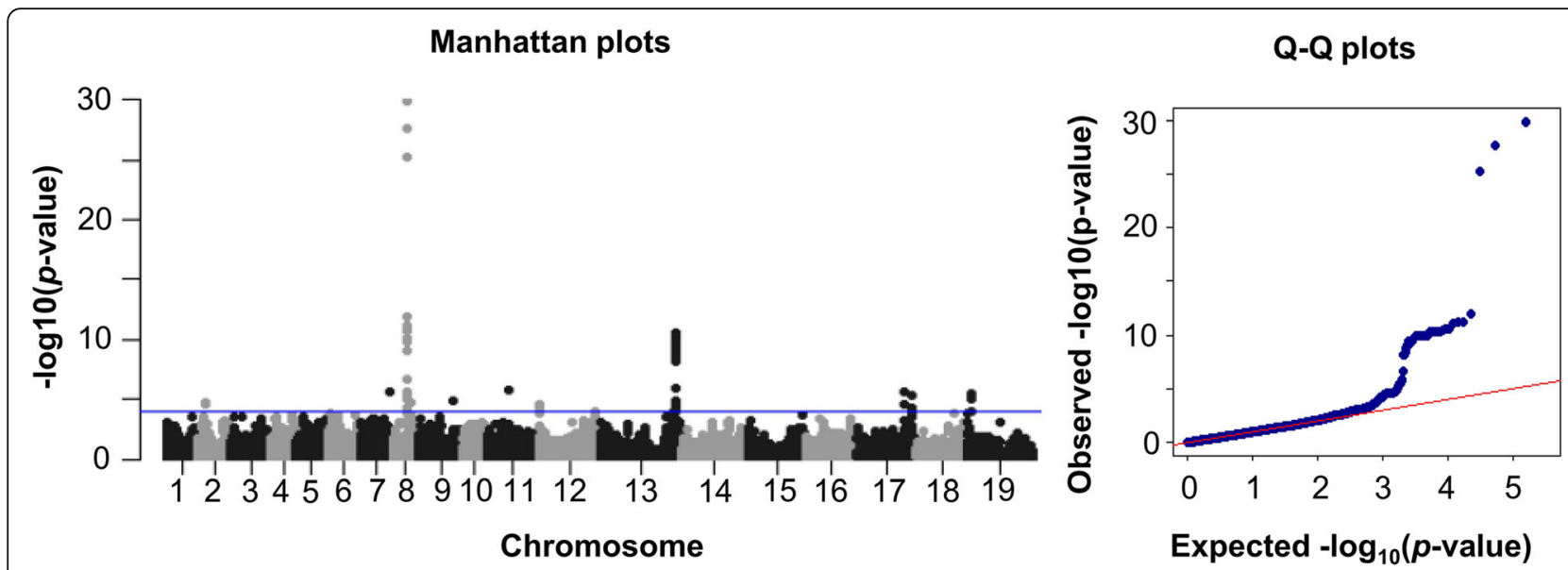

Fig. 2 Manhattan and quantile-quantile (QQ) plots of MLM showing genome-wide marker-trait associations for oleic acid content in 203 Chinese semiwinter rapeseed accessions grown in three different environments. The $-\log _{10}{ }^{(p)}$ significance threshold of 4 is indicated with a horizontal blue line

ECI1-HAP2 (t-test and mean value; Fig. 4b and c; Additional file 3: Table S3).

A similar analysis conducted in the C02 chromosome region, haplotype block (997,000-1,277,438 bp; C02_ Hap) was found to be significantly associated with oleic acid content (Fig. 3). This haplotype region contains three B. napus orthologues of the Arabidopsis genes FATTY ACID DESATURASE 8 (BnFAD8-C02; BnaC02g02240D) and SUGAR-DEPENDENT1 (BnSDP1-C02; BnaC02g02780D). FAD8 is involved in the fatty acid biosynthesis process, and SDP1 encodes a triacylglycerol lipase that is involved in the triglyceride metabolic process (Additional file 2: Table S2). In the C02_Hap region, combined whole-genome sequencing analysis of 50 accessions detected 9 SNPs located in these two gene regions, including six and three in the BnFAD8-C02 and BnSDP1-C02 regions, respectively (Additional file 6: Figure S1A). By comparing the oleic acid content and gene expression levels of the three haplotype alleles in the C02_Hap region, we found that C02_FAD8 + SDP1-Hap1 was associated with a higher oleic acid content than C02_FAD8 + SDP1-Hap2 and C02_FAD8 + SDP1-Hap3 and that BnFAD8-C02 and BnSDP1-C02 downregulated C02_FAD8 + SDP1-Hap1 expression in a manner that was related to higher oleic acid content accumulation ( $\mathrm{t}$-test and mean value; Additional file 6: Figure S1B and C; Additional file 3: Table S3).

\section{Co-expression network of genes from the haplotype regions}

To provide additional context for the proposed functions of BnMTACP2-A02, BnABCI13-A02 and BnECI1-A02 from the A02_Hap region and BnFAD8-C02 and BnSDP1-C02 from the C02_Hap region, we constructed a co-expression network for these five genes using gene expression data from siliques of Chinese semi-winter rapeseed accessions. The networks of the three and two genes from the A02_Hap and C02_Hap regions, respectively, were relatively independent (Additional file 8: Figure S3A). Then, we performed GO pathway analysis to uncover genes co-expressed with BnMTACP2-A02, BnABCI13-A02 and BnECI1-A02 in the A02_Hap region and BnFAD8-C02 and BnSDP1-C02 in the C02_Hap region. We found that these regions were significantly enriched in genes involved in lipid metabolic processes, fatty acid metabolic processes, acyl glycerol metabolic processes and so on (Additional file 7: Figure S2).

Based on the functional annotation, we further classified genes co-expressed with BnMTACP2-A02, BnABCI13-A02, and BnECI1-A02 from the A02_Hap region. These three candidate genes showed close correlations in the co-expression network (Fig. 5; Additional file 4: Table S4). In these three candidate gene subnetworks, 7 (27\%), 3 (12\%), 5 (11\%) and 2 (8\%) genes were lipid-related, fatty acid-related, glycerolrelated and carbohydrate-related, respectively. Another two clusters contained 12 (27\%), 8 (18\%) and 4 (9\%) genes involved in lipid-related, fatty acid-related and photosynthesis processes, respectively (Fig. 5; Additional file 4: Table S4). These results showed that BnMTACP2$\mathrm{A} 02$, BnABCI13-A02 and BnECI1-A02 from the A02 Hap region were interrelated with co-expression network genes that affected oleic acid content accumulation in rapeseed.

In the C02_Hap region, a similar gene co-expression network was constructed following this method. The candidate gene BnSDP1-C02 was indirectly related to the candidate gene BnFAD8-C02 in this network. In this co-expression network, a total of 14 genes were 


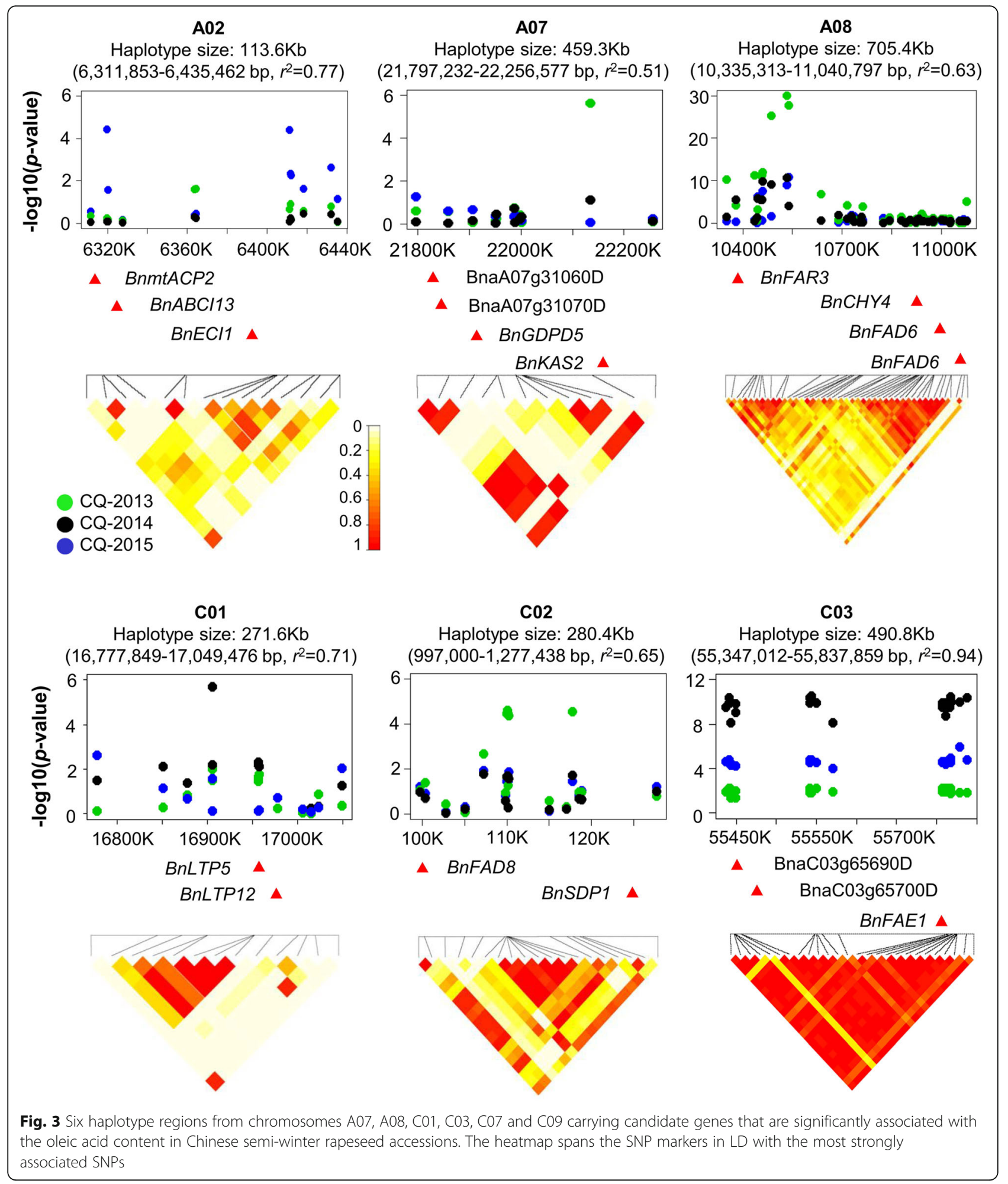

indirectly/directly associated with the candidate genes BnSDP1-C02 and BnFAD8-C02, including five and three genes involved in lipid and fatty acid metabolic processes, respectively (Additional file 8: Figure S3B; Additional file 4: Table S4)

\section{Discussion}

Selection of high-quality rapeseed with increased oil content and improved edible oils with a modified fatty acid composition has always been an important breeding goal. Strong selection has caused the formation of highly 


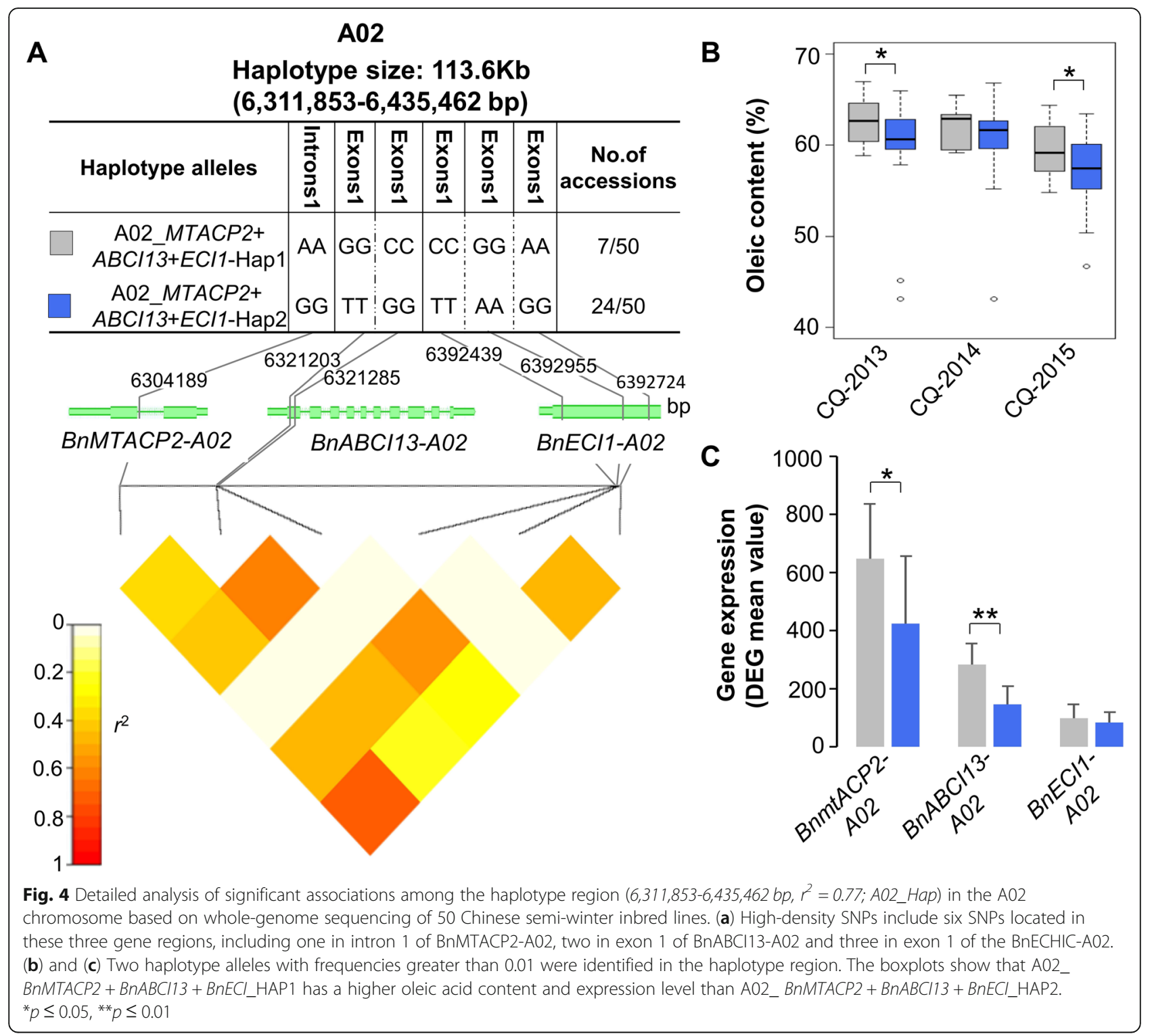

conserved haplotypes that harbor agronomically major and minor genes in QTL regions [19, 20]. With the advent of high-throughput SNP genotyping, genome-wide panels of SNPs allow haplotype-based GWASs to explore several causal genes if they are in close linkage and are associated with the complex traits of interest. In this study, we performed a genome-wide analysis of haplotype blocks associated with the oleic acid content. We identified six haplotypes that were significantly associated with the oleic acid content, each of which contained at least two genes related to lipid transport, fatty acid metabolism or glycerol metabolism. Fatty acid compositions are typical quantitative traits that are controlled by polygenic inheritance. We performed a preliminary screen of at least 2000 genes related to the fatty acid/ lipid metabolic processes in the whole genome by annotation analysis of the B. napus Darmor-bzh reference genome (data not shown). A recent study suggests that QTL regions containing many important genes involved in the pathway of fatty acid/lipid metabolismrelated to seed fatty acid composition in Brassica napus [21]. Indeed, strong selection has created haplotype regions carrying fatty acid metabolism genes in major QTLs for the erucic acid content on chromosomes A08 and $\mathrm{C} 03$ [22].

Generally, complex traits in crops show polygenic/ multilocus quantitative inheritance. Selection causes multiple interacting genes/alleles to change in the same fitness direction, at a similar evolutionary rate, and across the same time scale, to achieve a common phenotypic outcome. The haplotype-based analyses can capture potential interactions between SNPs/genes at a 


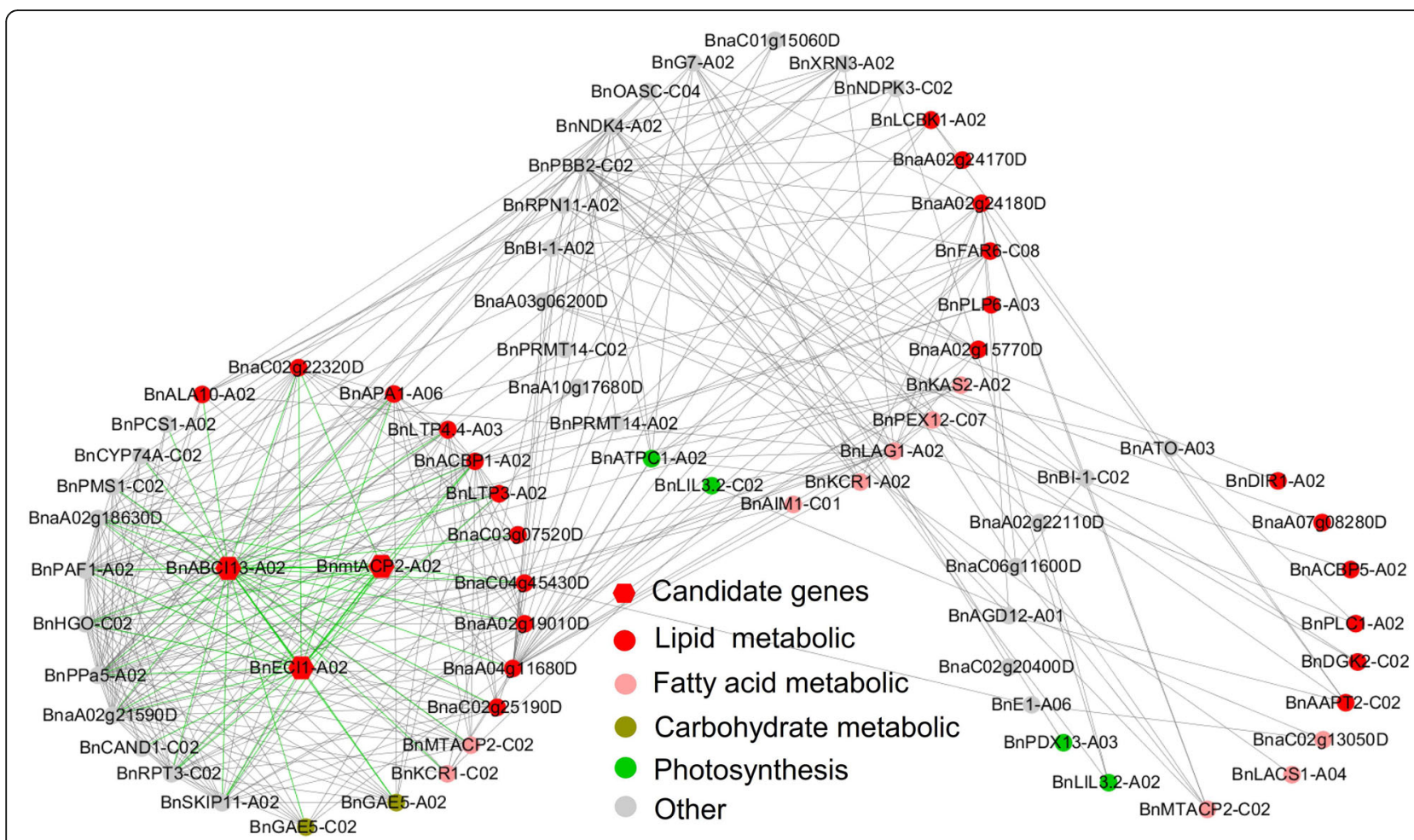

Fig. 5 Co-expression network analysis of three candidate genes from the A02_Hap region. Red pentagon nodes represent the candidate genes BnaMTACP2-A02, BnaABC113-A02 and BnaECHIC-A02. Based on the functional annotation, the co-expression network of these three candidate genes was classified into the following groups: lipids (red nodes), fatty acids (light pink nodes), glycerol (light goldenrod nodes), carbohydrates (yellow nodes), photosynthesis (green nodes) and others (grey nodes)

locus that affect phenotypic variation [23, 24]. Qian et al. [25] suggested that the haplotype region carrying several causal genes related to photosynthesis, chlorophyll degradation, flavonoid and proline biosynthesis processes that forms a potential interaction adjust in rapeseed growth and adaptation. Our results suggested that strong selection has caused close linkages among MTACP2A02, ABCI13-A02, and ECI1-A02 in the A02_Hap region, with positive effects on the accumulation of oleic acid content. MTACP2 encodes a member of the mitochondrial acyl carrier protein (ACP) family that is involved in the fatty acid biosynthesis process [26]. $\mathrm{ABCI} 13$ is an ATP-binding cassette $(\mathrm{ABC})$ transporter that is involved in chloroplast lipid import [27]. In plants, de novo fatty acid synthesis occurs in the plastid stroma, where acyl chains grow attached to the acyl carrier protein $(\mathrm{ACP})$ and become available for lipid assembly mainly in the form of C16:0-and C18:1-ACP [4, 28]. All acyl chains are made in plastids and assembled either in the plastids or the endoplasmic reticulum; thus, they require fatty acid/lipid transport [29]. ABC transporters direct participation in lipid or fatty acid/acyl-CoA transport. De Marcos Lousa et al. [30] suggested that functional and physical interactions between the $A B C$ transporter and the peroxisomal chain acyl-CoA synthetases were adjusted during the fatty acid/lipid metabolic processes. ECI1 encodes a peroxisomal delta3, delta2-enoyl CoA isomerase that is involved in fatty acid beta-oxidation [31]. Fatty acid beta-oxidation is an important catabolic process that is required for generation of acetyl-CoA and entry into the citric acid cycle. In plants, this process occurs predominantly within the peroxisomes, and therefore fatty acyl-CoAs must be imported from the cytosol by $\mathrm{ABC}$ transporters. These results suggest that $A B C$ transporters participate in the fatty acid synthesis/beta-oxidation process. Interestingly, we also detected a direct correlation among MTACP2$\mathrm{A} 02, \mathrm{ABCI} 13-\mathrm{A} 02$, and ECI1-A02 in the co-expression network. These three genes also directly or indirectly correlated with fatty acid synthesis/beta-oxidation, lipid transfer, carbohydrate and photosynthesis genes in the network adjusted for the fatty acid metabolic process (Fig. 5).

A similar result was produced in the Hap_C02 region, which contained two genes (FAD8-C02 and SDP1-C02) with close linkage that affected phenotypic variation in the seed oleic acid content. FAD8 was located in the chloroplast that is involved in the unsaturated fatty acid biosynthetic process [32]. Unsaturated fatty acids in seed lipids are catalyzed by fatty acid desaturases (FADs), 
FAD6 converts oleic acid (C18:1) to linoleic acid (C18: 2), and FAD7/FAD8 catalyzes the conversion of linoleic acid (C18:2) to linolenic acid (C18:3). Li et al. [33] suggested that a higher temperature caused a significant reduction in the FAD7/FAD8 expression level, which would increase oleic acid (C18:1) and linoleic acid (C18:2) accumulation and reduce the linolenic acid (C18:3) content. Drastic changes in temperature result in plant adaptation, with modified polyunsaturated fatty acid concentrations in their membranes and storage lipids. SDP1 limits triacylglycerol accumulation in vegetative tissues of Arabidopsis [34]. Fan et al. [35] suggested that SDP1 played key roles in diverting fatty acids from membrane lipid synthesis toward $\beta$-oxidation through a transient triacylglycerol pool. Kim et al. [36] suggested that SDP1 deficiency generated by RNAi technology produced a notable increase in seed oil accumulation. Our result showed that BnFAD8-C02 and BnSDP1-C02 expression was downregulated in accessions with higher oleic acid contents. Meanwhile, the co-expression network directs correlations of BnPLIP3-C08 with BnFAD8-C02, BnSDP1-C02, BnGED1-C02, BnMED15_1, BnLRSC05, and BnPRMT4B-C05, which include five genes involved in fatty acid synthesis/beta-oxidation (Additional file 8: Figure S3). These results suggest that strong selection has caused the genes of fatty acid synthesis/ beta-oxidation pathways being in a potential interaction and co-regulation for the accumulation of oleic acid in rapeseed.

Various arguments advocating haplotype-based analysis rather than single-marker analysis have been proposed. Haplotype analyses also provide important insights into the history of both natural and artificial selection (breeding) and can give valuable guidance to breeders seeking to diversify crop gene pools. Crop breeding programs aim to improve populations by increasing the frequency of favorable alleles of traits. The identification of shifts in allele frequencies within the genome can be important information for breeders, since it alerts them to monitor specific haplotype alleles and can be used to design appropriate breeding strategies [37]. Recently, some research has shown favorable haplotype alleles related to improved cold tolerance in rice [38], short stature or long inflorescence branches in sorghum [39] and drought tolerance in maize [40]. We also reveal the beneficial haplotype alleles $\mathrm{A}_{2} 2_{-}$ MTACP2 + ABCI13 + ECI1-Hap1 and C02_FAD8 + SDP1-Hap1, which contribute to high oleic acid content. This finding will provide an opportunity to combine these beneficial haplotype alleles and further improve the oleic acid content in rapeseed.

\section{Conclusion}

The high-throughput genome-wide single nucleotide polymorphic (SNP) markers have been successfully used in association mapping for the dissection of complex agronomic traits. Moreover, these markers allow wholegenome scans to identify haplotype regions that are significantly correlated with complex traits variation. In this study, GWAS in combination with haplotype analysis has identified six haplotype regions significantly associated with oleic acid (C18:1) that mapped to chromosomes A02, A07, A08, C01, C02, and C03. In addition, whole-genome sequencing of 50 rapeseed lines revealed three genes in the A02 chromosome haplotype region and two genes in the $\mathrm{C} 02$ chromosome haplotype region that were closely linked to the oleic acid content phenotypic variation. These results will help to develop functional haplotype markers for the improvement of the oleic acid content in rapeseed.

\section{Methods \\ Plant material and phenotypic data}

A diverse panel of 203 homozygous Chinese semi-winter inbred lines broadly encompassed the allelic variability in the Asian B. napus gene pool. The materials (Additional file 1: Table S1) were obtained as self-pollinated seeds from Southwest University, Chongqing, China, where they represent part of a breeding program spanning genetic diversity from the broader Asian gene pool. This population has been used previously in genomic diversity analysis [22] and GWAS of the chlorophyll content [22]. Field trials were conducted by applying an unreplicated complete randomized design in Chongqing in 2013, 2014 and 2015 (designated CQ-2013, CQ-2014, and CQ-2015, respectively). Each line was grown in a three-row plot with 10 homozygous inbred plants in each row. Self-pollinated seeds were collected from each line. About $5 \mathrm{~g}$ of dry mature seeds of each line (selected three strains, and each strain measured two times) were analyzed for oleic acid (C18:1) content by near-infrared spectroscopy (NIRS; Foss NIR Systems Inc., USA), each line selected three strains, and each strain measured two times.

The phenotypic analysis results, including the phenotypic distribution, mean value, standard deviation, correlation coefficient, and minimum and maximum values of oleic acid from the 203 accessions, were calculated and analyzed with the R packages Hmisc [41] and Psych [42]. Variance components were estimated via restricted maximum likelihood- using the statistical software package SPSS Statistics for Windows Version 22.0 (IBM Corp., Armonk, NY, USA), and broad-sense heritability $\left(\mathrm{H}^{2}\right)$ for the oleic acid content was calculated, across the environments and in each environment, using the following equation [43]: 


$$
\mathrm{H}^{2}=\frac{\sigma_{\mathrm{g}}^{2}}{\sigma_{\mathrm{g}}^{2}+\sigma_{\mathrm{e}}^{2} / n}
$$

where the genotypic and residual variance components are represented by $\sigma_{\mathrm{g}}^{2}$ and $\sigma_{\mathrm{e}}^{2}$, respectively, and estimates of the residual variance were divided by the number (environments) $n$.

\section{Genotypic data}

The 203 accessions were genotyped using the Brassica $60 \mathrm{~K}$ Illumina $^{\circ}$ Infinium SNP array (IlluminaInc., San Diego, CA, USA) by TraitGenetics GmbH (Gatersleben, Germany). We applied a filtering for single-copy BLAST hits that were physically assignable to the B. napus "Darmor-bzh" reference genome assembly version 4.1 [44] (http://www.genoscope.cns.fr/brassicanapus/data/). Only markers that exhibited at least $95 \%$ sequence identity and no gaps in their 50-bp probe sequence were retained, resulting in 28,698 unique, locus-specific SNPs. In an additional preprocessing step, all markers with more than $10 \%$ missing values and a minor allele frequency below $5 \%$ were excluded. A total of 24,338 highquality, single-locus, SNP markers with a minor allele frequency (MAF) $\geq 0.05$ were used for the GWAS (Additional file 5: Table S5).

\section{Genome-wide association analysis}

A detailed description of the genetic composition and population structure of the diversity set was provided by Qian et al. [22]. Relative kinship analysis was performed using the TASSEL 5.0 software [45]. Marker-trait associations were examined by applying the TASSEL 5.0 software with the $\mathrm{Q}+\mathrm{K}$ mixed linear model as proposed by $\mathrm{Yu}$ et al. [46]. The mixed linear model was as follows:

$$
\mathrm{y}=\mu+\mathrm{S} \alpha+\mathrm{Pv}+\mathrm{Zu}+\mathrm{e}
$$

The model was used to test associations between the SNPs and phenotypes, where y is the vector of phenotypic observations, $\mu$ is the overall mean, $\alpha$ is a vector of the fixed allelic effects associated with the SNP under investigation, $\mathrm{v}$ is a vector of fixed population effects, $\mathrm{u}$ is a vector of random genetic background effects, and e is the vector of residuals. The matrix $P$ contains information on population structure, using the first five principal components as fixed factors to adjust the model for population stratification, while $\mathrm{S}$ and $\mathrm{Z}$ are incidence matrices relating $\mathrm{y}$ to $\mathrm{a}$ and $\mathrm{u}$, respectively. The variances of the random effects $\mathrm{u}$ and $\mathrm{e}$ are assumed to be normally distributed with $\mathrm{u} \sim N\left(0, \mathrm{G} \sigma_{\mathrm{g}}^{2}\right)$ and $e \sim N(0$, $\mathrm{R} \sigma_{\mathrm{g}}^{2}$ ), where $G$ is a $203 \times 203$ genomic relationship matrix and $R$ is a $203 \times 203$ matrix in which the offdiagonal elements are 0 and the diagonal elements are the reciprocal of the number of phenotypic observations per individual. The $\mathrm{G}$ matrix was computed according to the first method proposed by VanRaden et al. [47]. Since every genotype was tested only once per environment and GWAS were performed for all the environmenttrait combinations separately, $\mathrm{R}$ corresponds to the identity matrix I.

The observed $P$ values from the marker-trait associations were used to display $\mathrm{Q}-\mathrm{Q}$ and Manhattan plots using the R package qqman [48]. The critical $P$-value for assessing the significance of SNP-trait associations was calculated separately for the oleic acid content based on the false discovery rate (FDR) [49]. An FDR $<0.05$ was used to identify significant associations with the oleic acid content at a cut-off value of $-\log _{10}{ }^{(P)}=4$.

\section{0 accession resequencing analysis}

Genomic DNA was extracted from fresh leaves of each plant using the cetyltrimethyl ammonium bromide (CTAB) method and sequenced using the Illumina Hiseq $^{\text {ti }} 4000$ (Illumina Co., Inc., San Diego, CA, USA) with a $5 \mathrm{X}$ sequencing depth and 125-bp paired-end sequencing length. Library preparation and sequencing were carried out at the Biomarker Technologies Corporation (Beijing, China). SNPs detected among the accessions that have described by Dong et al. [50]. SNPs with a missing call rate $>0.6$ were excluded. The remaining SNPs with missing call rates below 0.6 were filled using the software "beagle" v4.1 [51] (https://faculty.washington.edu/browning/beagle/beagle.html). SNP loci with a heterozygous rate greater than $25 \%$ and a MAF less than 0.05 were removed. Ultimately, we obtained 532,005 high-quality SNPs that were used to analysis LD among candidate genes on significant association haplotype region.

\section{Phenotypic correlations with haplotype diversity groups}

Significant haplotype blocks were identified using the $\mathrm{R}$ package LD heatmap [52], with haplotypes defined across regions of homozygous markers with $\operatorname{LD}\left(\mathrm{r}^{2}\right)>$ 0.50 between the first and last markers in the block. Haplotype alleles with a frequency $>0.01$ were used for the comparative phenotype analysis. A two-sample t-test (assuming unequal variance) was used to test for significant phenotypic differences in the oleic acid content among the haplotype alleles.

\section{Gene content in the haplotype block regions}

Annotated genes within the oleic acid content-associated haplotype regions were extracted from the $B$. napus Darmor-bzh reference genome v. 4.1 [44]. To verify the most likely gene functions, we determined annotations of the closest orthologous Arabidopsis thaliana genes by BLAST matching against the Arabidopsis genome database (http://www.arabidopsis.org/). 


\section{Transcriptome sequencing}

Thirteen of 50 resequencing accessions were selected for the sampling of siliques for transcriptome analysis fifteen days after pollination. These 13 accessions were selected based on different oil contents (i.e. 4 accessions with lower oil content, 4 with medium oil content and 5 with higher oil content). The siliques were immediately frozen in liquid nitrogen and stored at $-80^{\circ} \mathrm{C}$ prior to $R N A$ extraction. At least $3 \mu \mathrm{g}$ of total RNA was used for each accession to construct paired-end sequencing libraries according to the manufacturer's instructions (Illumina Inc.). Paired-end reads (125 bp) were evaluated using the Illumina HiSeq 2500 platform. The raw reads were filtered to remove sequencing adapters and low-quality reads $(>10 \%$ unknown bases or $>50 \%$ of bases with a quality $<10)$ and generate "clean" reads that were subsequently aligned to the Darmor-bzh reference genome v.4.1 [44] using HISAT 2 (http://ccb.jhu.edu/software/ hisat/index.shtml). The gene expression levels were calculated using HTSeq 0.6.1 [53] (https://pypi.python.org/ pypi/HTSeq). To screen differentially expressed genes (DEGs), we used a q-value $<0.05$ and an absolute value of $\log 2$ (fold change) normalized to $>1$ based on the $R$ package DEGSeq v1.22.0 [54].

\section{Co-expression analysis}

The co-expression edges were calculated, and a soft threshold value of 0.9 was chosen in the WGCNA R package [55]. Genes with Pearson Correlation Coefficients (PCCs) greater than or equal to 0.55 were used for co-expression network construction by CYTOSCAPE3.6 [56].

\section{Supplementary information}

Supplementary information accompanies this paper at https://doi.org/10. 1186/s12864-020-6711-0.

Additional file 1 Table S1. Source, population structure, 50 resequencing accessions, 13 transcriptomes accessions, heritability and oleic acid phenotype information of 203 Chinese semi-winter rapeseed accessions.

Additional file $\mathbf{2}$ Table S2. SNPs and gene information in the significant association of haplotype region.

Additional file $\mathbf{3}$ Table S3. Detailed analysis of two haplotype regions from chromosomes A02 and C02, including SNPs, genes and haplotype alleles related to oleic acid content variation in 50 resequencing accessions.

Additional file 4 Table S4. Gene information of co-expression network in $\mathrm{A} 02$ and $\mathrm{C} 02$ chromosome haplotype region.

Additional file $\mathbf{5}$ Table S5. Flanking oligonucleotide sequences, chromosomal positions (best BLAST hit) and genotype calls for the 24,338unique polymorphic SNPS used for the chromosome structure and LD analyses in the diversity panel of 203 B. napus inbred lines.

Additional file 6 Figure S1. Detailed analysis of the significant associations of the haplotype region (997,000-1,277,438 bp; C02_Hap) in chromosome $\mathrm{C} 02$ by whole-genome sequencing of 50 Chinese semiwinter inbred lines. (A) A total of 21 SNPs were located in these three gene regions, including six and three in the BnFAD8-CO2 and BnSDP1-CO2 gene regions, respectively. (B) and (C) Three haplotype alleles with frequencies greater than 0.01 were identified in the haplotype region. The boxplots show that C02_BnFAD8 + BnSDP1_HAP1 has a higher oleic acid content and lower expression level than C02 BnFAD8 + BnSDP1_HAP3. ${ }^{*} p \leq 0.05,{ }^{* *} p \leq 0.01,{ }^{* * *} p \leq 0.01$.

Additional file 7 Figure S2. GO pathway of three and two candidate genes from the A02_Hap and C02_Hap region co-expression networks, respectively.

Additional file 8 Figure S3. Co-expression networks of three and two candidate genes from the A02_Hap and $\mathrm{CO} 2$ _Hap regions, respectively. (A) Red pentagon nodes represent candidate genes in the A02-Hap and C02-Hap regions. Green and blue nodes represent genes that are directly and indirectly co-expressed with the candidate genes in these haplotype regions, respectively. (B) Co-expression network of two candidate genes from the C02_Hap region. Red pentagon nodes represent the candidate genes BnFAD8-CO2 and BnSDP1-C02. Based on the functional annotation, these two candidate gene expression network were classified into the following groups: lipid (red nodes), fatty acids (lightpink nodes) and others (grey nodes).

\section{Abbreviations}

GWAS: Genome-wide association study; LD: Linkage disequilibrium; QTLs: Quantitative trait locus; SNP: Single nucleotide polymorphism; ACP: Acyl carrier protein; ABC: ATP-binding cassette; DEGs: Differentially expressed genes; PCCs: Pearson correlation coefficients; FDR: False discovery rate; MAF: Minor allele frequency; WGCNA: Weighted Gene Co-Expression Network Analysis

\section{Acknowledgments}

Not applicable.

\section{Authors' contributions}

$\mathrm{WH}, \mathrm{RS}$, and CG coordinated and designed the study. LQ and MG wrote the manuscript. MY, CW and QZ performed data mining, bioinformatics. YC, $X H$, $Z Z, H C$, and WL carried out reagents and the field experiments. HJ, KV, and ZL contributed to interpretation and modification of the data and manuscript. All authors have read and approved the final manuscript.

\section{Funding}

This study was funded by the Hunan Province Science and Technology Plan (grant no. 2014FJ1000) to CG and the Hunan Provincial Key Research and Development Program (grant no. 2016JC2024) to HC.

\section{Availability of data and materials}

All data generated and results analyzed during this study are included in this article and its supplementary information. Transcriptome data used for coexpression network and gene expression analysis in Additional file 4: Table S4. 24,338 high-quality SNP markers for GWAS in Additional file 5: Table S5. Resequencing 50 accessions of Chinese semi-winter rapeseed from NCBI under BioProject accession PRJNA358784.

Ethics approval and consent to participate

Not applicable.

Consent for publication

Not applicable.

\section{Competing interests}

The authors declare that they have no competing interests.

\section{Author details}

${ }^{1}$ Collaborative Innovation Center of Grain and Oil Crops in South China, Hunan Agricultural University, Changsha 410128, China. ${ }^{2}$ College of Horticulture and Landscape Architecture, Southwest University, Chongqing 400715, China. ${ }^{3}$ Precision Medicine Lab, Rehman Medical Institute (RMI), Phase 5, Hayatabad, Peshawar 25000, Pakistan. ${ }^{4}$ Queensland Alliance for Agriculture and Food Innovation, The University of Queensland, St Lucia, QLD, Australia. ${ }^{5}$ The Roslin Institute University of Edinburgh Easter Bush Research Centre Midlothian, Edinburgh EH25 9RG, UK. 'Department of Plant 
Breeding, IFZ Research Centre for Biosystems, Land Use and Nutrition, Justus Liebig University, Heinrich-Buff-Ring 26-32, 35392 Giessen, Germany. 'Oil Crops Research Institute of the Chinese Academy of Agricultural Sciences, Key Laboratory of Biology and Genetic Improvement of Oil Crops, Ministry of Agriculture, Wuhan 430062, China.

\section{Received: 12 June 2019 Accepted: 31 March 2020}

Published online: 23 April 2020

\section{References}

1. Napier JA, Haslam RP, Beaudoin F, Cahoon EB. Understanding and manipulating plant lipid composition: metabolic engineering leads the way. Curr Opin Plant Biol. 2014;19:68-75.

2. Menendez JA, Lupu R. Mediterranean dietary traditions for the molecular treatment of human cancer: anti-oncogenic actions of the main olive oil's monounsaturated fatty acid oleic acid. Curr Pharm Biotechnol. 2006;7:495-502.

3. Pinzi S, Garcia IL, Lopez-Gimenezm FJ, et al. The ideal vegetable oil-based biodiesel composition: a review of social: economical and technical implications. Energy Fuel. 2009;23:2325-41.

4. Li-Beisson Y, Shorrosh B, Beisson F, et al. Acyl-lipid metabolism. Arabidopsis Book. 2010;8:e0133.

5. Wang $X$, Long $Y$, Yin $Y$, et al. New insights into the genetic networks affecting seed fatty acid concentrations in Brassica napus. BMC Plant Biol. 2015;15:91.

6. Javed N, Geng J, Tahir M, et al. Identification of QTL influencing seed oil content fatty acid profile and days to flowering in Brassica napus L. Euphytica. 2016;207:191-211.

7. Yan X, Li J, Wang R, et al. Mapping of QTLs controlling content of fatty acid composition in rapeseed (Brassica napus). Genes Genom. 2011;33:365-71.

8. Zhao J, Dimov Z, Becker HC, et al. Mapping QTL controlling fatty acid composition in a doubled haploid rapeseed population segregating for oil content. Mol Breeding. 2008;21:115-25.

9. Burns MJ, Barnes SR, Bowman JG, et al. QTL analysis of an intervarietal set of substitution lines in Brassica napus: (i) seed oil content and fatty acid composition. Heredity. 2003;90:39-48.

10. Smooker AM, Wells $R$, Morgan C, et al. The identification and mapping of candidate genes and QTL involved in the fatty acid desaturation pathway in Brassica napus. Theor Appl Genet. 2011;122:1075-90.

11. Hu X, Sullivan-Gilbert M, Gupta M, Thompson SA. Mapping of the loci controlling oleic and linolenic acid contents and development of fad 2 and fad3 allele-specific markers in canola (Brassica napus L.). Theor Appl Genet. 2006;113:497-507.

12. Qu C, Jia L, Fu F, et al. Genome-wide association mapping and identification of candidate genes for fatty acid composition in Brassica napus L using SNP markers. BMC Genomics. 2017;18:232.

13. Guan $M$, Huang $X$, Xiao Z, et al. Association mapping analysis of fatty acid content in different ecotypic rapeseed using mrMLM. Front Plant Sci. 2019 9:1872.

14. Voss-Fels KP, Qian L, Parra-Londono S, et al. Linkage drag constrains the roots of modern wheat. Plant Cell Environ. 2017:40:717-25.

15. Qian L, Qian W, Snowdon RJ. Haplotype hitchhiking promotes trait coselection in Brassica napus. Plant Biotechnol J. 2016;14:1578-88.

16. Contreras-Soto RI, Mora F, de Oliveira MA, et al. Genome-wide association study for agronomic traits in soybean using SNP markers and SNP-based haplotype analysis. PLoS One. 2017:12:e0171105.

17. Schaefer RJ, Michno JM, Jeffers J, et al. Integrating co-expression networks with GWAS to prioritize causal genes in maize. Plant Cell. 2018;30:2922-42.

18. Zhang J, Yang Y, Zheng K, et al. Genome-wide association studies and expression-based quantitative trait loci analyses reveal roles of HCT2 in caffeoylquinic acid biosynthesis and its regulation by defense-responsive transcription factors in Populus. New Phytol. 2018;220:502-16.

19. Voss-Fels K, Snowdon RJ. Understanding and utilizing crop genome diversity via high-resolution genotyping. Plant Biotechnol J. 2016;14:1086-94.

20. Xie W, Wang G, Yuan M, et al. Breeding signatures of rice improvement revealed by a genomic variation map from a large germplasm collection. Proc Natl Acad Sci U S A. 2015;112:E5411-9.

21. Chen F, Zhang W, Yu K, et al. Unconditional and conditional QTL analyses of seed fatty acid composition in Brassica napus L. BMC Plant Biol. 2018;18:49.

22. Qian L, Qian W, Snowdon RJ. Sub-genomic selection patterns as a signature of breeding in the allopolyploid Brassica napus genome. BMC Genomics. 2014;15:1170.
23. Snowdon RJ, Abbadi A, Kox T, Schmutzer T, Leckband G. Heterotic haplotype capture: precision breeding for hybrid performance. Trends Plant Sci. 2015;20:410-3.

24. Jiang Y, Schmidt RH, Reif JC. Haplotype-based genome-wide prediction models exploit local epistatic interactions among markers. G3. 2018:8:168799.

25. Qian L, Voss-Fels K, Cui Y, et al. Deletion of a stay-green gene associates with adaptive selection in Brassica napus. Mol Plant. 2016:9:1559-69.

26. Meyer EH, Heazlewood JL, Millar AH. Mitochondrial acyl carrier proteins in Arabidopsis thaliana are predominantly soluble matrix proteins and none can be confirmed as subunits of respiratory complex I. Plant Mol Biol. 2007; 64:319-27.

27. Lu B, Xu C, Awai $K$, Jones AD, Benning C. A small ATPase protein of Arabidopsis, TGD3, involved in chloroplast lipid import. J Biol Chem. 2007; 282:35945-53.

28. Troncoso-Ponce MA, Nikovics K, Chloe M, et al. New insights on the organization and regulation of the et al. new insights on the organization and regulation of the fatty acid biosynthetic network in the model higher plant Arabidopsis thaliana. Biochimie. 2015;120:3-8.

29. Li N, Xu C, Li-Beisson Y, Philippar K. Fatty acid and lipid transport in plant cells. Trends Plant Sci. 2016:21:145-58.

30. De Marcos LC, van Roermund CW, Postis VL, et al. Intrinsic acyl-CoA thioesterase activity of a peroxisomal ATP binding cassette transporter is required for transport and metabolism of fatty acids. Proc Natl Acad Sci U S A. 2013;110:1279-84

31. Goepfert S, Vidoudez C, Tellgren-Roth C, et al. Peroxisomal Delta (3), Delta (2)-enoyl CoA isomerases and evolution of cytosolic paralogues in embryophytes. Plant J. 2008;56:728-42.

32. Li SS, Wang LS, Shu QY, et al. Fatty acid composition of developing tree peony (Paeonia section Moutan DC.) seeds and transcriptome analysis during seed development. BMC Genomics. 2015;16:208.

33. Li Q, Zheng Q, Shen W, Cram D, Fowler DB, Wei Y, Zou J. Understanding the biochemical basis of temperature-induced lipid pathway adjustments in plants. Plant Cell. 2015;27:86-103.

34. Kelly AA, Erp van H, Quettier AL, et al. The sugar-dependent1 lipase limits triacylglycerol accumulation in vegetative tissues of Arabidopsis. Plant Physiol 2013; 162:1282-1289.

35. Fan J, Yan C, Roston R, Shanklin J, Xu C. Arabidopsis lipins, PDAT1 acyltransferase, and SDP1 triacylglycerol lipase synergistically direct fatty acids toward $\beta$-oxidation, thereby maintaining membrane lipid homeostasis. Plant Cell. 2014:26:4119-34.

36. Kim MJ, Yang SW, Mao HZ, et al. Gene silencing of Sugar-dependent 1 (JCSDP1), encoding a patatin-domain triacylglycerol lipase, enhances seed oil accumulation in Jatropha curcas. Biotechnol Biofuels. 2014;7:36.

37. Collard BC, Mackill DJ. Marker-assisted selection: an approach for precision plant breeding in the twenty-first century. Philos Trans R Soc Lond Ser B Biol Sci. 2008:363:557-72.

38. Zhang Z, Li J, Pan Y, et al. Natural variation in CTB4a enhances rice adaptation to cold habitats. Nat Commun. 2017:8:14788.

39. Morris GP, Ramu P, Deshpande SP, et al. Population genomic and genomewide association studies of agroclimatic traits in sorghum. Proc Natl Acad Sci U S A. 2012;110:453-8.

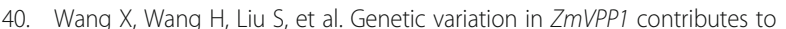
drought tolerance in maize seedlings. Nat Genet. 2016:48:1233-41.

41. Harrell F. Hmisc: Harrell Miscellaneous. R package version 4.1-1. https:// CRAN.R-project.org/package=Hmisc. 2018.

42. Revelle W. Psych: Procedures for Personality and Psychological Research. Evanston. $\mathrm{R}$ package version $=1.8 .4$. https: $/ /$ CRAN.R-project.org $/$ package $=$ psych: Northwestern University; 2018.

43. Bekele WA, Fiedler K, Shiringani A, Schnaubelt D, Windpassinger S, Uptmoor $\mathrm{R}$, et al. Unravelling the genetic complexity of sorghum seedling development under low-temperature conditions. Plant Cell Environ. 2014;37: 707-23.

44. Chalhoub B, Denoeud F, Liu S, et al. Early allopolyploid evolution in the post-Neolithic Brassica napus oilseed genome. Science. 2014;345:950-3.

45. Bradbury PJ, Zhang Z, Kroon DE, et al. TASSEL: software for association mapping of complex traits in diverse samples. Bioinformatics. 2007;23:26335.

46. Yu J, Pressoir G, Briggs WH. A unified mixed-model method for association mapping that accounts for multiple levels of relatedness. Nat Genet. 2006; 38:203-8. 
47. VanRaden PM. Efficient methods to compute genomic predictions. J Dairy Sci. 2008;91:4414-23.

48. Turner SD. QQman: an R package for visualizing GWAS results using Q-Q and Manhattan plots. J Open Source Softw. 2018:3:731.

49. Benjamini $Y$, Hochberg $Y$. Controlling the false discovery rate: a practical and powerful approach to multiple testing. JR Stat Soc B. 1995;57:289-300.

50. Dong H, Tan C, Li Y, et al. Genome-wide association study reveals both overlapping and independent genetic loci to control seed weight and silique length in Brassica napus. Front Plant Sci. 2018;9:921.

51. Browning SR, Browning BL. Rapid and accurate haplotype phasing and missing-data inference for whole-genome association studies by use of localized haplotype clustering. Am J Hum Genet. 2007;31:365-75.

52. Shin JH, Blay S, McNeney B, Graham J. LDheatmap: an R function for graphical display of pairwise linkage disequilibria between single nucleotide polymorphisms. J Stat Soft. 2006;16:1-9.

53. Anders S, Pyl PT, Huber W. HTSeq - a Python framework to work with highthroughput sequencing data. Bioinformatics. 2015;31:166-9.

54. Wang L, Feng Z, Wang X, Wang X, Zhang X. DEGseq: an R package for identifying differentially expressed genes from RNA-seq data. Bioinformatics. 2010;26:136-8.

55. Langfelder P, Horvath S. WGCNA: an R package for weighted correlation network analysis. BMC Bioinformatics. 2008;9:559.

56. Smoot ME, Ono K, Ruscheinski J, Wang PL, Ideker T. Cytoscape 2.8: new features for data integration and network visualization. Bioinformatics. 2010; $27: 431-2$.

\section{Publisher's Note}

Springer Nature remains neutral with regard to jurisdictional claims in published maps and institutional affiliations.

Ready to submit your research? Choose BMC and benefit from:

- fast, convenient online submission

- thorough peer review by experienced researchers in your field

- rapid publication on acceptance

- support for research data, including large and complex data types

- gold Open Access which fosters wider collaboration and increased citations

- maximum visibility for your research: over $100 \mathrm{M}$ website views per year

At BMC, research is always in progress.

Learn more biomedcentral.com/submissions 